\title{
Experimental Verification of Infinity Norm Approach for Force Maximization of Manipulators Driven by Bi-articular Actuators
}

\author{
Valerio Salvucci, Yasuto Kimura, Sehoon Oh and Yoichi Hori
}

\begin{abstract}
Recently, there has been increasing attention on animal inspired manipulators equipped with bi-articular actuators. Bi-articular actuated manipulators usually present more actuators than joints, resulting in actuator redundancy. In order to resolve this torque load sharing problem, in this paper a new approach based on infinity norm is proposed and successfully implemented on BiWi, Bi-articularly actuated and Wire driven manipulator.

The infinity norm model maximizes the force at the end effector given the maximum actuator joint torques. Therefore, it is an approach to optimize actuators design for bi-articularly actuated manipulators. The infinity norm approach is based only on linear functions for actuator redundancy resolution.
\end{abstract}

\section{INTRODUCTION}

Manipulators presenting animal musculo-skeletal characteristics such as bi-articular actuators have been proposed for more than two decades [1]. Recently, there has been increasing attention on such biologically inspired manipulators, both in hardware and control design aspects [2] [3] [4].

A manipulator based on such a model usually has more actuators than joints, resulting in actuator redundancy. In order to resolve this torque load sharing problem many approaches have been proposed.

Several animal inspired approaches to solve actuator redundancy as fatigue minimization [5], muscle force minimization [6] [7], total muscle metabolic energy consumption [8], total muscle stress minimization [9] [10] have been proposed. These approaches are mainly based on a phenomenological nature, which can make an implementation on robotics applications difficult.

Pseudo-inverse matrices are often used for kinematics redundancy resolution of manipulators [11]. This approach is also used to resolve actuator redundancy [12]. MoorePenrose is the simplest pseudo-inverse matrix, and corresponds to the minimization of the 2 -norm (euclidean norm) [13].

In this paper, our new approach to resolve actuator redundancy based on infinity norm [14] is experimentally verified by using Biwi, Bi-articularly actuated and Wire driven manipulator. The $\infty-$ norm approach maximizes the force at the end effector given the maximum actuator joint torques. Therefore, it is used as an approach to optimize actuators

This work is supported by Inamori Foundation

V. Salvucci, S. Oh, Y. Hori with Department of Electrical Engineering University of Tokyo, 3-7-1 Hongo, Bunkyoku Tokyo, 1130033, Japan \{valerio, sehoon\}@hori.k.u-tokyo.ac.jp horiek.u-tokyo.ac.jp

Y. Kimura is with Department of Advanced Energy Engineering, The University of Tokyo 5-1-5 Kashiwanoha, Kashiwa, Chiba, 277-8561, Japan kimura@hori.k.u-tokyo.ac.jp design for bi-articularly actuated manipulators. Moreover, the $\infty-$ norm approach is based only on linear functions to resolve actuator redundancy.

The $\infty$-norm approach is compared with the MoorePenrose pseudo-inverse approach $(2-$ norm $)$ in terms of maximum output force at the end effector by using both numerical calculation and experimental results. Under the same maximum actuator joint torques, the maximum output force at the end effector calculated using the proposed $\infty-$ norm approach is greater than the one obtained using $2-$ norm approach. For the experimental results, BiWi, Biarticularly actuated and Wire driven manipulator is proposed.

In Section II, main features and statics of bi-articularly actuated manipulators are described. In Section III, 2 - norm and $\infty-$ norm approaches for actuator redundancy resolution are introduced. In Section IV, Biwi, Bi-articularly actuated and Wire driven manipulator is described together with the feedforward control strategy used in the experiment. In Section V, the proposed $\infty$-norm approach is compared with 2-norm approach in terms of maximum output force at the end effector. Finally, in Section VI, the advantages of the $\infty$-norm approach are summarized. The proof of the closed form solution of the $\infty-$ norm approach is reported in Appendix I.

\section{CHARACTERISTICS AND MODELING OF BI-ARTICULAR ACTUATED MANIPULATORS}

In conventional manipulators each joint is driven by one actuator. On the contrary, animal limbs present a complex musculo-skeletal structure based on two types of muscles:

1) Mono-articular muscles, which produce a torque on one joint.

2) Multi-articular muscles, which produce torque on two or more consecutive joints at the same time. Gastrocnemius is a bi-articular muscle in the human leg.

A simplified model of the complex animal musculo-skeletal system is shown in Fig. 1. This model is based on 6 contractile actuators - extensors $\left(e_{1}, e_{2}\right.$ and $\left.e_{3}\right)$ and flexors $\left(f_{1}, f_{2}\right.$ and $\left.f_{3}\right)$ - coupled in three antagonistic pairs.

- $e_{1}-f_{1}$ and $e_{2}-f_{2}$ : pairs of mono-articular actuators which produce torques about joints 1 and 2, respectively.

- $e_{3}-f_{3}$ : pair of bi-articular actuators which produce torque about joints 1 and 2 contemporaneously.

The statics of the arm driven by bi-articular actuators of Fig. 1 are shown in Fig. 2 where:

- $T_{1}$ and $T_{2}$ are total torques at joints 1 and 2 , respectively. 


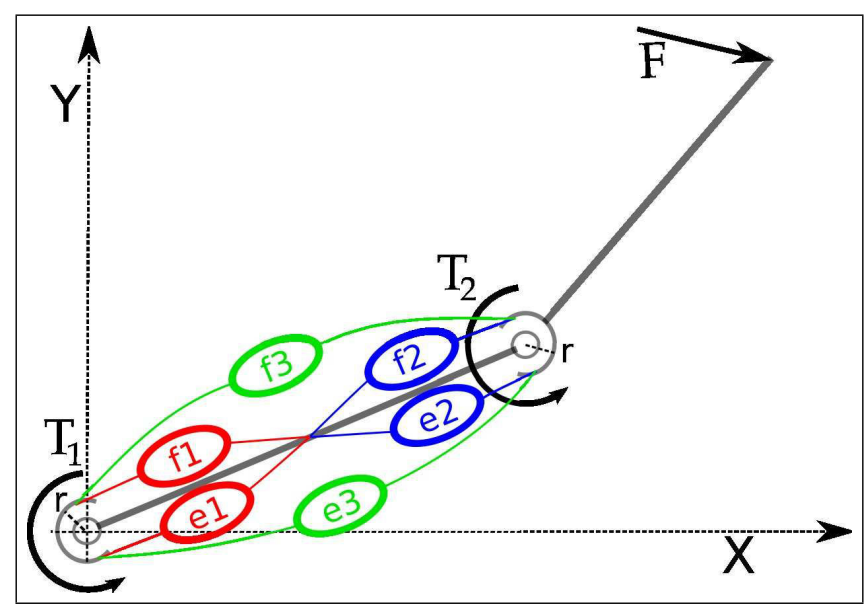

Fig. 1. Scheme a two-link arm with 4 mono- and 2 bi-articular actuators

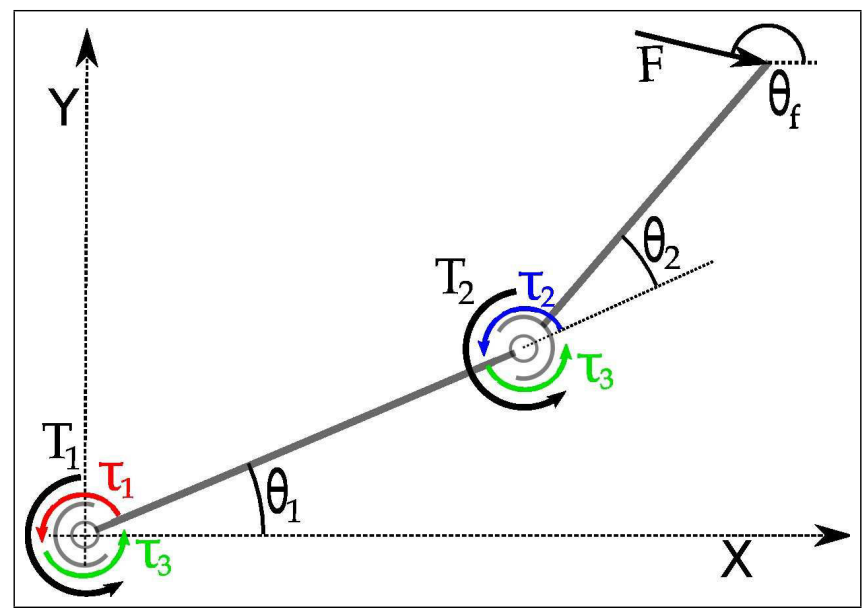

Fig. 2. Statics of a two-link arm with mono and bi-articular actuators

- $\tau_{1}$ and $\tau_{2}$ are torques produced by mono-articular actuators at joints 1 and 2 respectively, calculated as:

$$
\begin{aligned}
& \tau_{1}=\left(f_{1}-e_{1}\right) r \\
& \tau_{2}=\left(f_{2}-e_{2}\right) r
\end{aligned}
$$

where $r$ is the distance between the joint axis and the point where the force is applied.

- $\tau_{3}$ is the bi-articular torque produced at both joints:

$$
\tau_{3}=\left(f_{3}-e_{3}\right) r
$$

- $\mathbf{F}$ is a general force at the end effector.

The statics of this system are therefore expressed by:

$$
\left\{\begin{array}{l}
T_{1}=\tau_{1}+\tau_{3} \\
T_{2}=\tau_{2}+\tau_{3}
\end{array}\right.
$$

Manipulators equipped with bi-articular actuators have numerous advantages: dramatical increase in range of end effector impedance that can be achieved without feedback [1], realization of path tracking and disturbance rejection using just feedforward control [12], improvement of balance control for jumping robots that do not use force sensors

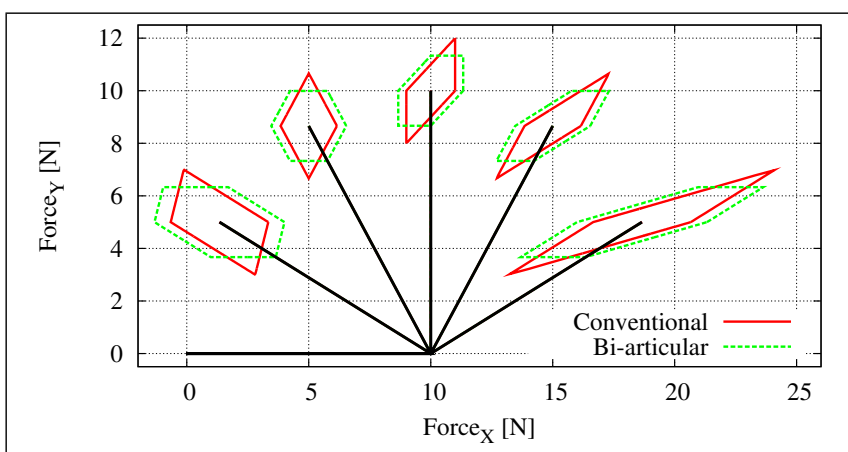

Fig. 3. Maximum output force at the end effector for conventional and arm driven by bi-articular actuators

[15]. Moreover, multi-joints actuators such as tri-articular actuators, increase the efficiency in force production [16].

Another advantage of arm equipped with bi-articular actuators is the ability to produce a maximum output force at the end effector in a more homogeneously distributed way [17]. In Fig. 3 the maximum output force at the end effector for a two-link traditional manipulator and a robot arm equipped with bi-articular actuators is shown for comparison. In the case of traditional manipulator, two actuators with maximum joint torque $T_{1}=T_{2}=10 \mathrm{Nm}$ are considered. On the other hand, for the bi-articular actuators driven arm three actuators with maximum joint torque $\tau_{1}=\tau_{2}=\tau_{3}=6.66 \mathrm{Nm}$ are taken into account. Therefore, the total maximum actuator torque in the two cases is the same. The conventional quadrilateral shape becomes an hexagon for arms driven by bi-articular actuators that therefore produce a maximum force at the end effector more homogeneously distributed in respect to output force direction. This aspect is peculiar for applications which interact with humans such as rehabilitation robots, as well as for jumping and waking robots [4] [15].

\section{APPROACHES FOR ACTUATOR REDUNDANCY RESOLUTION}

A two-link manipulator with the statics shown in Fig. 2 is driven at least by three actuators, resulting in actuator redundancy. Given $\tau_{1}, \tau_{2}$ and $\tau_{3}$, it is possible to determine $\mathbf{T}=\left[T_{1}, T_{2}\right]^{T}$, and so $\mathbf{F}=\left[F_{x}, F_{y}\right]^{T}$ by using the transpose Jacobian:

$$
\left[\begin{array}{l}
T_{1} \\
T_{2}
\end{array}\right]=J^{T}\left[\begin{array}{l}
F_{x} \\
F_{y}
\end{array}\right]
$$

where

$$
J=\left[\begin{array}{cc}
-l_{1} \sin \left(\theta_{1}\right)-l_{2} \sin \left(\theta_{1}+\theta_{2}\right) & -l_{2} \sin \left(\theta_{1}+\theta_{2}\right) \\
l_{1} \cos \left(\theta_{1}\right)+l_{2} \cos \left(\theta_{1}+\theta_{2}\right) & l_{2} \cos \left(\theta_{1}+\theta_{2}\right)
\end{array}\right]
$$

$F_{x}$ and $F_{y}$ are the orthogonal projection of $\mathbf{F}$ on the $\mathrm{x}$ axis and y-axis, respectively. On the other hand, given $\mathbf{F}$, and therefore $\mathbf{T}$, it is generally not possible to determine uniquely $\tau_{1}, \tau_{2}$ and $\tau_{3}$ (see (4)).

In the following two approaches to resolve actuator redundancy $-2-$ norm and $\infty-$ norm - are described. It is assumed that $\tau_{1}^{\max }=\tau_{2}^{\max }=\tau_{3}^{\max }$, where $\tau_{i}^{\max }(i=1,2,3)$ is the maximum joint torque that the actuator $i$ produces. 


\section{A. 2-norm approach}

Given the desired joint torques $T_{1}$ and $T_{2}$, three actuators joint torques $\tau_{1}, \tau_{2}$ and $\tau_{3}$ are calculated using 2 -norm by solving the following problem:

$$
\begin{array}{ll}
\text { minimize } & \sqrt{\left(\tau_{1}\right)^{2}+\left(\tau_{2}\right)^{2}+\left(\tau_{3}\right)^{2}} \\
\text { subject to } & \left\{\begin{array}{l}
T_{1}=\tau_{1}+\tau_{3} \\
T_{2}=\tau_{2}+\tau_{3}
\end{array}\right.
\end{array}
$$

The solution of the problem is:

$$
\left\{\begin{array}{l}
\tau_{1}=\frac{2}{3} T_{1}-\frac{1}{3} T_{2} \\
\tau_{2}=-\frac{1}{3} T_{1}+\frac{2}{3} T_{2} \\
\tau_{3}=\frac{1}{3} T_{1}+\frac{1}{3} T_{2}
\end{array}\right.
$$

\section{B. $\infty$-norm approach}

Given the desired joint torques $T_{1}$ and $T_{2}$, three actuators joint torques $\tau_{1}, \tau_{2}$ and $\tau_{3}$ are calculated using $\infty-$ norm by solving the following problem:

$$
\begin{array}{ll}
\text { minimize } & \max \left\{\left|\tau_{1}\right|,\left|\tau_{2}\right|,\left|\tau_{3}\right|\right\} \\
\text { subject to } & \left\{\begin{array}{l}
T_{1}=\tau_{1}+\tau_{3} \\
T_{2}=\tau_{2}+\tau_{3}
\end{array}\right.
\end{array}
$$

A closed form solution of the problem is determined on the basis of the values of $T_{1}$ and $T_{2}$ as follows:

- if $T_{1} T_{2} \leq 0$

$$
\left\{\begin{array}{l}
\tau_{1}=\frac{T_{1}-T_{2}}{T_{2}^{2}} \\
\tau_{2}=\frac{T_{2}-T_{1}}{\tau_{1}^{2}} \\
\tau_{3}=\frac{T_{1}+T_{2}}{2}
\end{array}\right.
$$

- if $T_{1} T_{2}>0$ and $\left|T_{1}\right| \leq\left|T_{2}\right|$

$$
\left\{\begin{array}{l}
\tau_{1}=T_{1}-\frac{T_{2}}{2} \\
\tau_{2}=\frac{T_{2}}{2} \\
\tau_{3}=\frac{T_{2}}{2}
\end{array}\right.
$$

- if $T_{1} T_{2}>0$ and $\left|T_{1}\right|>\left|T_{2}\right|$

$$
\left\{\begin{array}{l}
\tau_{1}=\frac{T_{1}}{2} \\
\tau_{2}=T_{2}-\frac{T_{1}}{2} \\
\tau_{3}=\frac{T_{1}}{2}
\end{array}\right.
$$

Proof of (12), (13), and (14) is reported in Appendix I. It is trivial to verify that (12), (13), and (14) are continuous in all the domain $D=\left\{T_{1}, T_{2}\right\}$.

Given a generic force at the end effector $\mathbf{F}$, the actuators inputs $\tau_{1}, \tau_{2}$ and $\tau_{3}$ are calculated in the following way.

1) Calculate the joint torques by using (5), $\mathbf{T}=J^{T} \mathbf{F}$.

2) According to calculated $T_{1}$ and $T_{2}$, the desired actuators inputs are directly determined using linear equations:

- if $T_{1} T_{2} \leq 0$ use (12)

- if $T_{1} T_{2}>0$ and $\left|T_{1}\right| \leq\left|T_{2}\right|$ use (13)

- if $T_{1} T_{2}>0$ and $\left|T_{1}\right|>\left|T_{2}\right|$ use (14)

Therefore, the proposed $\infty-$ norm approach is based on the manipulator Jacobian to determine the required joint torques, and uses only linear functions to resolve actuator redundancy.

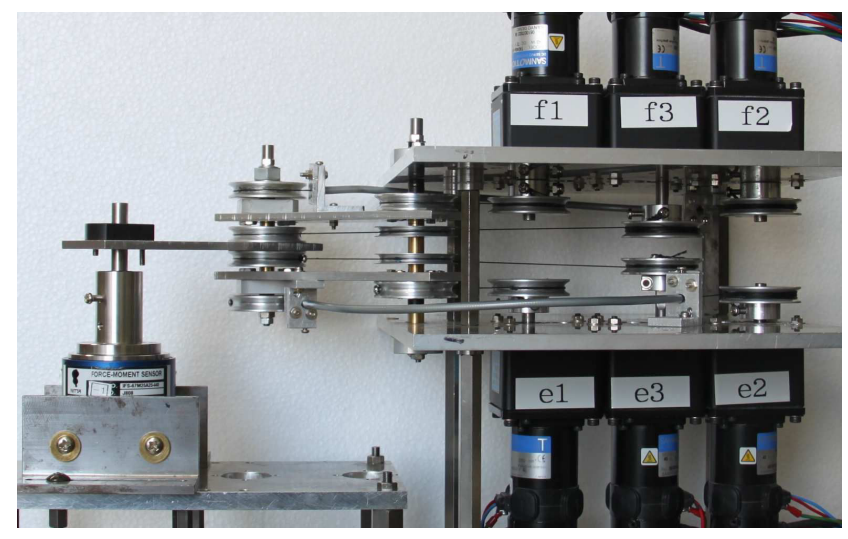

Fig. 4. BiWi:Bi-articularly actuated and Wire driven manipulator

TABLE I

BIWI CHARACTERISTICS

\begin{tabular}{|c|c|}
\hline Parameter & value \\
\hline Link 1 = Link 2 & $112[\mathrm{~mm}]$ \\
Pulleys diameter (all) & $44[\mathrm{~mm}]$ \\
Thrust wire & $30[\mathrm{~mm}]$ \\
\hline
\end{tabular}

TABLE II

ACTUATOR AND SENSOR SYSTEM

\begin{tabular}{|c|c|}
\hline Motors & Sanyo T404-012E59 \\
\hline Servo system & TS1A02AA \\
\hline Force sensor & Nitta IFS-67M25A15-I40 \\
\hline
\end{tabular}

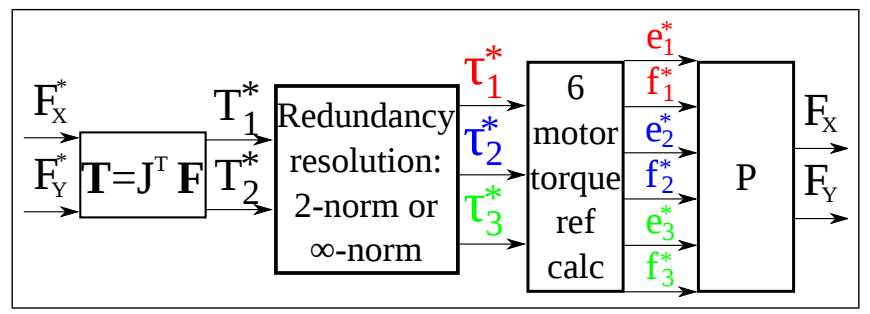

Fig. 5. Feedforward control block diagram

\section{EXPERIMENTAL SET-UP}

BiWi, Bi-articularly actuated and Wire driven manipulator, is shown in Fig. 4. BiWi has 6 motors, each representing one of the muscles in Fig. 1. The power is transmitted to the joints through pulleys and polyethylene wires. Basic characteristics of BiWi and of the actuator and sensor systems are shown in Tab. I and Tab. II, respectively. Further characteristics of BiWi are described in [18].

The feedforward control block diagram used to collect the data is shown in Fig. 5. $F_{x}^{*}$ and $F_{y}^{*}$ are the desired force at the end effector. $J$ is the manipulator Jacobian. $\tau_{1}^{*}, \tau_{2}^{*}$ and $\tau_{3}^{*}$ are the desired actuator joint torques as in Fig. 2, which are calculated using $2-$ norm or $\infty-$ norm approach from the desired joint torques $T_{1}^{*}$ and $T_{2}^{*} \cdot t_{1}^{*}-t_{6}^{*}$ are the 6 actuator joint torque reference. They are calculated as:

$$
e_{1}^{*}=\left\{\begin{array}{cl}
\tau_{1}^{*} & \text { if } \tau_{1}^{*}<0 \\
0 & \text { otherwise }
\end{array}\right.
$$




$$
\begin{aligned}
& f_{1}^{*}=\left\{\begin{array}{cc}
\tau_{1}^{*} & \text { if } \tau_{1}^{*}>0 \\
0 & \text { otherwise }
\end{array}\right. \\
& e_{2}^{*}=\left\{\begin{array}{cc}
K_{t l} \tau_{2}^{*} & \text { if } \tau_{2}^{*}<0 \\
0 & \text { otherwise }
\end{array}\right. \\
& f_{2}^{*}=\left\{\begin{array}{cc}
K_{t l} \tau_{2}^{*} & \text { if } \tau_{2}^{*}>0 \\
0 & \text { otherwise }
\end{array}\right. \\
& e_{3}^{*}=\left\{\begin{array}{cc}
\tau_{3}^{*} & \text { if } \tau_{3}^{*}<0 \\
0 & \text { otherwise }
\end{array}\right. \\
& f_{3}^{*}=\left\{\begin{array}{cc}
\tau_{3}^{*} & \text { if } \tau_{3}^{*}>0 \\
0 & \text { otherwise }
\end{array}\right.
\end{aligned}
$$

In order to compensate for the inevitable transmission loss in the thrust wires the reference motor torques for joint $2-$ $e_{2}^{*}$ and $f_{2}^{*}-$ are multiplied by a constant $K_{t l}=1.33$.

The end effector output force $(\mathbf{F})$ is measured by using a force sensor, and its steady state value is taken into account.

\section{RESULTS}

The $2-$ norm and the proposed $\infty-$ norm approaches are compared in terms of maximum output force at the end effector, under the same maximum actuator joint torque, that is $\tau_{1}^{\max }=\tau_{2}^{\max }=\tau_{3}^{\max }$.

The calculated and measured maximum output force at the end effector of BiWi, obtained using the $2-$ norm and $\infty-$ norm approaches, are shown in Fig. 6(a) for $\theta_{1}=-60.0^{\circ}$ and $\theta_{2}=120.0^{\circ}$, in Fig. 7(a) for $\theta_{1}=-45^{\circ}$ and $\theta_{2}=90^{\circ}$ and in Fig. 8(a) for $\theta_{1}=-30^{\circ}$ and $\theta_{2}=60^{\circ}$. The experimental results show that the measured force at the end effector agrees with the calculated one. Moreover, the maximum output force at the end effector is greater when using the $\infty-$ norm approach. The relative difference in maximum output force magnitude between the two approaches:

$$
F^{\text {diff }}=\frac{\left|F_{\infty-n}^{\max }\right|-\left|F_{2-n}^{\max }\right|}{\left|F_{2-n}^{\max }\right|}
$$

is shown in Fig. 6(b), in Fig. 7(b) and in Fig. 8(b) for the three configurations. In case of robot legs an output force with $\theta_{f}=0$ is perpendicular to the ground, an therefore important for standing, walking and jumping actions [15]. The output force for $\theta_{f}=0$ using $\infty-$ norm is about $30 \%$ higher than the one using 2 -norm.

The actuator joint torque patterns of two approaches are shown in Fig. 6(c), in Fig. 7(c) and in Fig. 8(c) for the three configurations. For both the approaches the actuator joint torque patterns are continuous in respect to the output force angle $\left(\theta_{f}\right.$ in Fig. 2). Hence, the 3 switching conditions used for the redundancy resolution in the $\infty-$ norm approach do not result in torque reference discontinuities, which could cause instability to the system.

\section{CONCLUSIONS}

In this paper, a new approach based on $\infty$-norm to resolve actuator redundancy for bi-articular actuated manipulators is proposed. A closed form solution for the proposed method is derived. The $\infty$-norm approach maximizes the force at the end effector given the maximum actuator joint torques. Therefore, it is an approach to optimize actuators

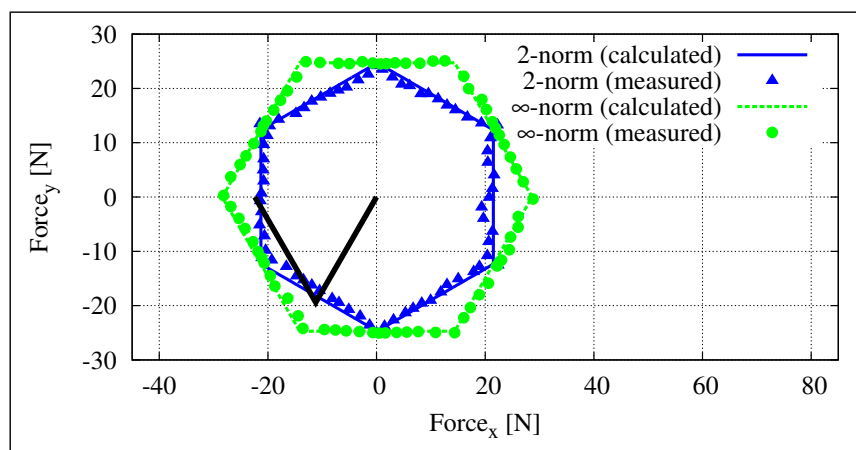

(a) Measured maximum output force

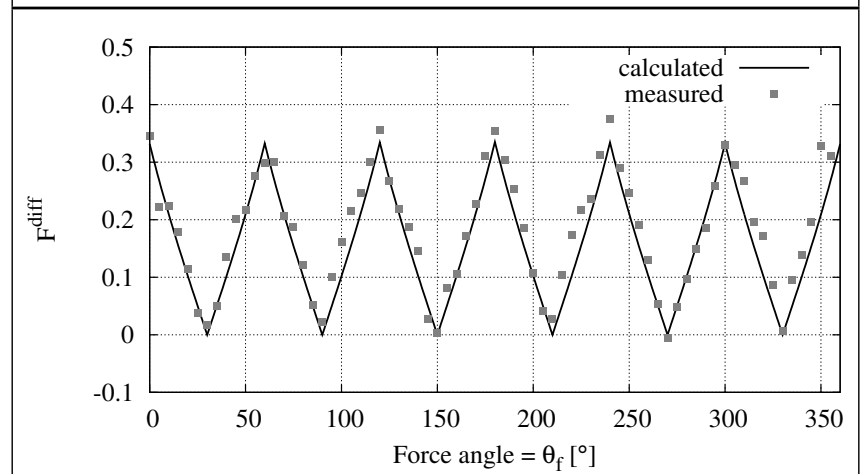

(b) Relative difference in maximum output force magnitude

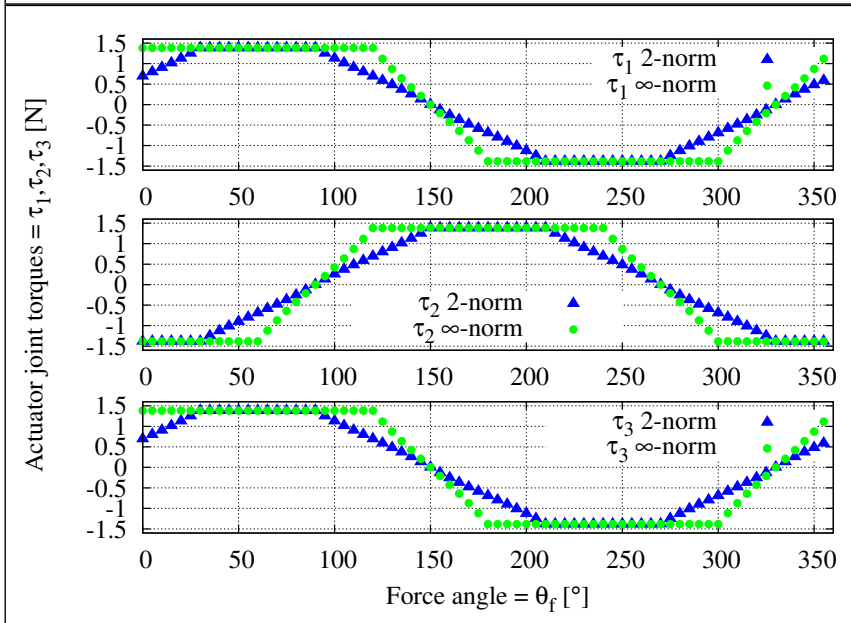

(c) Actuator joint torques

Fig. 6. $\theta_{1}=-60^{\circ}, \theta_{2}=120^{\circ}$

design for bi-articularly actuated manipulators. Moreover, only linear functions are used to resolve actuator redundancy.

The proposed approach is compared in terms of maximum output force at the end effector with Moore-Penrose pseudo-inverse model which is based on 2-norm. Under the same maximum actuator joint torques, the proposed approach allows to obtain a greater output force at the end effector (up to $30 \%$ ), especially in output force direction peculiar for applications which interact with humans such as rehabilitation robots, as well as for jumping/waking robots.

Biwi, Bi-articularly actuated and Wire driven manipulator, and a feedforward control strategy are used to experimentally validate the proposed $\infty-$ norm approach. 


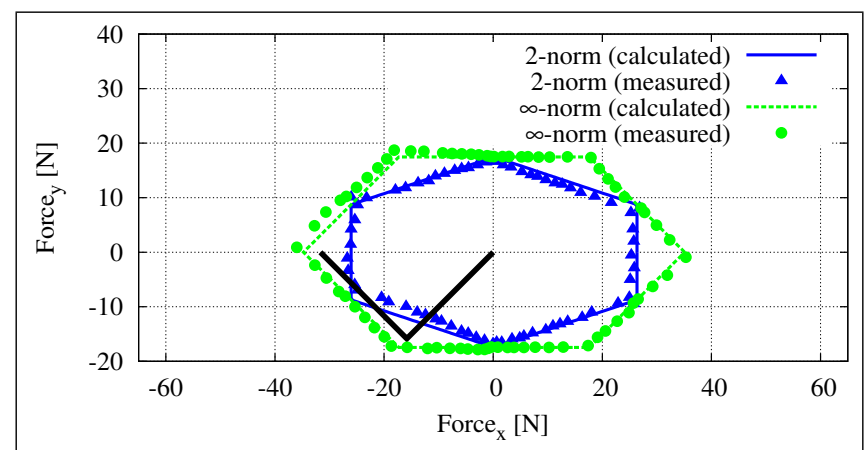

(a) Measured maximum output force

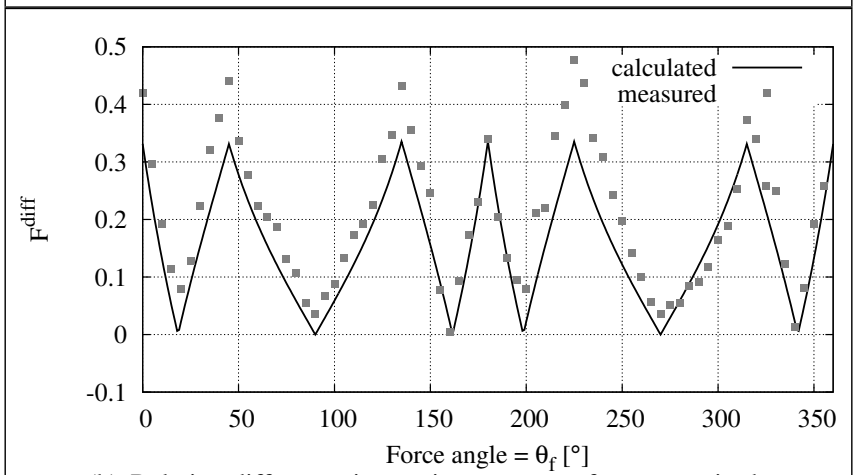

(b) Relative difference in maximum output force magnitude

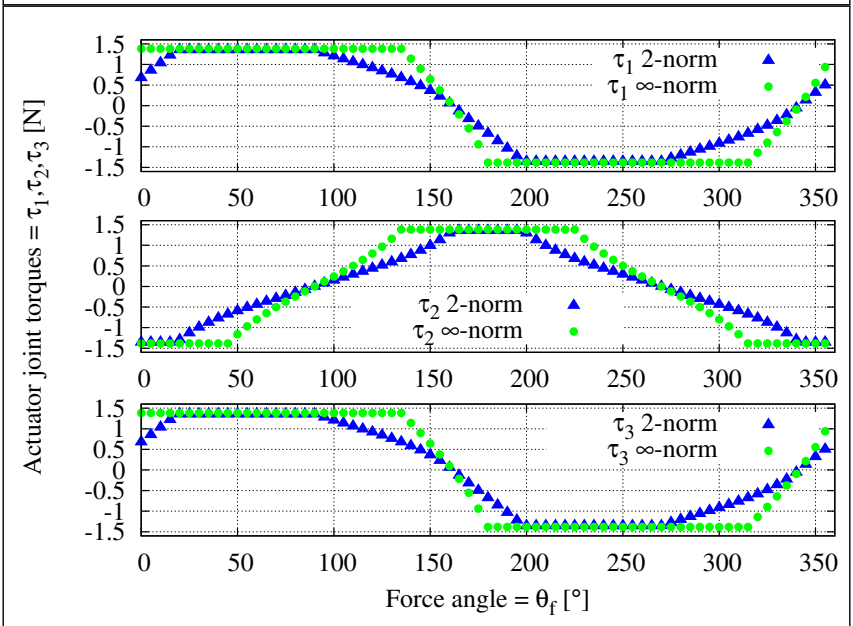

(c) Actuator joint torques

Fig. 7. $\theta_{1}=-45^{\circ}, \theta_{2}=90^{\circ}$

\section{APPENDIX I}

PROOF OF CLOSED FORM SOLUTION FOR THE $\infty-$ norm APPROACH

Actuator redundancy problem based on $\infty-$ norm approach expressed by (10) and (11) can be written for a simpler notation as:

$$
\begin{array}{ll}
\text { minimize } & \max \{|x|,|y|,|z|\} \\
\text { subject to } & \left\{\begin{array}{l}
T_{1}=x+z \\
T_{2}=y+z
\end{array}\right.
\end{array}
$$

where $k_{1}$ and $k_{2}$ are the joint torques $T_{1}$ and $T_{2}$, and $x, y, z$ are the actuator joint torques $\tau_{1}, \tau_{2}, \tau_{3}$, respectively. A closed

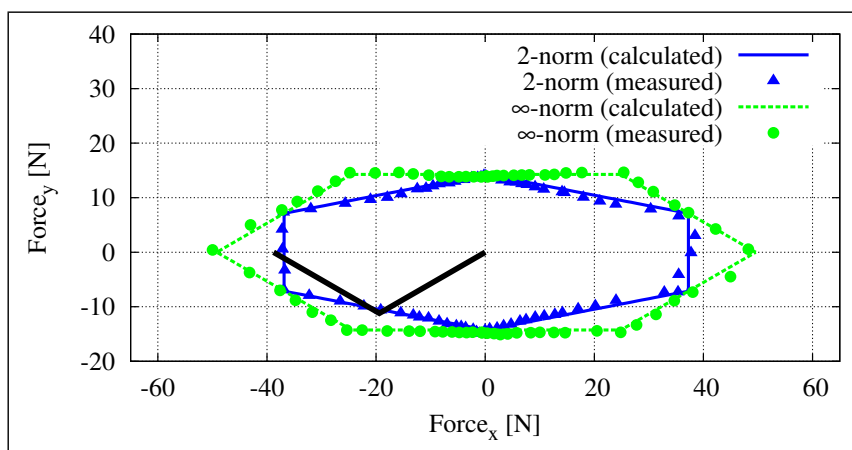

(a) Measured maximum output force

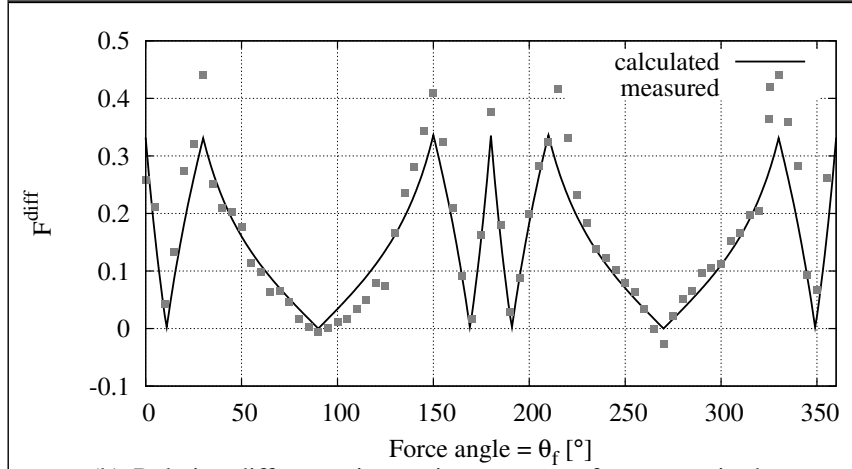

(b) Relative difference in maximum output force magnitude

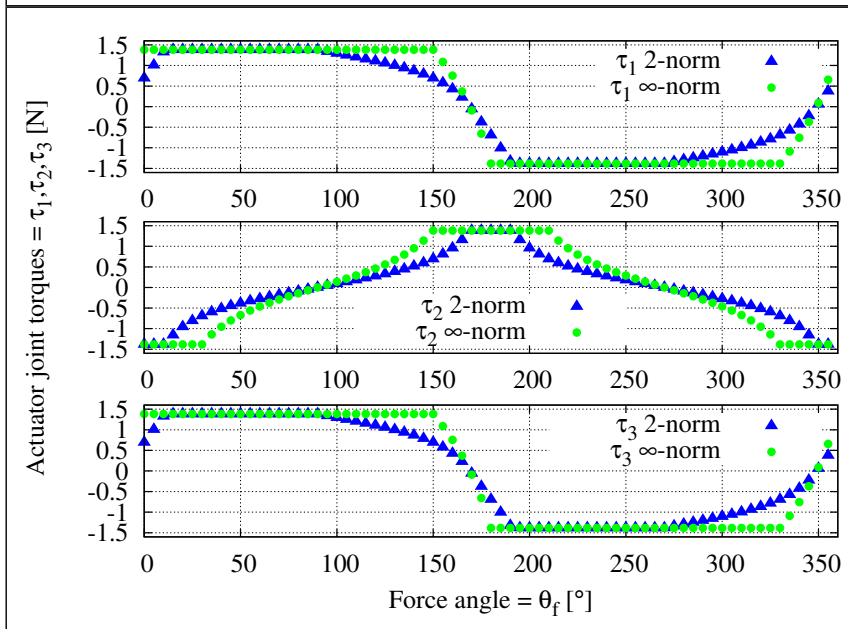

(c) Actuator joint torques

Fig. 8. $\theta_{1}=-30^{\circ}, \theta_{2}=60^{\circ}$

form solution is determined as in the following.

The searched solution has to satisfy at least one of the three equations $|x|=|y|,|y|=|z|,|x|=|z|$. In fact, when one of three variable's absolute value decreases at least one of the other two increases. Therefore, for any solution of the problem with $|x| \neq|y| \neq|z|$ it is possible to decrease the higher value among the three so to be equal to at least one of the other two. Therefore, the searched solution is one among the following 6 : 
1) $x=-y$ :

$$
\left\{\begin{array} { l } 
{ x + z = k _ { 1 } } \\
{ y + z = k _ { 2 } } \\
{ x = - y }
\end{array} \Rightarrow \left\{\begin{array}{l}
x=\frac{k_{1}-k_{2}}{2} \\
y=\frac{k_{2}-k_{1}}{2} \\
z=\frac{k_{1}+k_{2}}{2}
\end{array}\right.\right.
$$

2) $y=z$ :

$$
\left\{\begin{array} { l } 
{ x + z = k _ { 1 } } \\
{ y + z = k _ { 2 } } \\
{ y = z }
\end{array} \Rightarrow \left\{\begin{array}{l}
x=k_{1}-\frac{k_{2}}{2} \\
y=\frac{k_{2}}{2} \\
z=\frac{k_{2}}{2}
\end{array}\right.\right.
$$

3) $x=z$ :

$$
\left\{\begin{array} { l } 
{ x + z = k _ { 1 } } \\
{ y + z = k _ { 2 } } \\
{ x = z }
\end{array} \Rightarrow \left\{\begin{array}{l}
x=\frac{k_{1}}{2} \\
y=k_{2}-\frac{k_{1}}{2} \\
z=\frac{k_{1}}{2}
\end{array}\right.\right.
$$

4) $x=y$ :

$$
\left\{\begin{array}{l}
x+z=k_{1} \\
y+z=k_{2} \\
x=y
\end{array}\right.
$$

If $k_{1} \neq k_{2}$ there is no solution. If $k_{1}=k_{2}=k$ there are infinite solution and the best one is

$$
\left\{\begin{array}{l}
x=\frac{k}{2} \\
y=\frac{k}{2} \\
z=\frac{k}{2}
\end{array}\right.
$$

which is equal to (25) or (26).

5) $y=-z$ :

$$
\left\{\begin{array}{l}
x+z=k_{1} \\
y+z=k_{2} \\
y=-z
\end{array}\right.
$$

If $k_{2} \neq 0$ there is no solution. If $k_{2}=0$ there are infinite solution and the best one is

$$
\left\{\begin{array}{l}
x=\frac{k_{1}}{2} \\
y=-\frac{k_{1}}{2} \\
z=\frac{k_{1}}{2}
\end{array}\right.
$$

which is equal to (26).

6) $x=-z$;

$$
\left\{\begin{array}{l}
x+z=k_{1} \\
y+z=k_{2} \\
x=-z
\end{array}\right.
$$

If $k_{1} \neq 0$ there is no solution. If $k_{1}=0$ there are infinite solution and the best one is

$$
\left\{\begin{array}{l}
x=-\frac{k_{2}}{2} \\
y=\frac{k_{2}}{2} \\
z=\frac{k_{2}}{2}
\end{array}\right.
$$

which is equal to (25).

Therefore, the searched solution is (24) or (25) or (26).

Among the 3 possible solutions the searched one is directly selected on the basis of $k_{1}$ and $k_{2}$ as follows (the variable subscript represents the respective equation number):

- if $k_{1} k_{2} \leq 0$

$$
\left\{\begin{array}{l}
\left|z_{24}\right| \leq\left|x_{24}\right|=\left|y_{24}\right| \\
\left|x_{24}\right| \leq\left|x_{25}\right| \\
\left|y_{24}\right| \leq\left|y_{26}\right|
\end{array}\right.
$$

Therefore, solution is (24) which corresponds to (12). - if $k_{1} k_{2}>0$ and $\left|k_{1}\right| \leq\left|k_{2}\right|$

$$
\left\{\begin{array}{l}
\left|x_{25}\right| \leq\left|y_{25}\right|=\left|z_{25}\right| \\
\left|y_{25}\right| \leq\left|y_{26}\right| \\
\left|z_{25}\right| \leq\left|z_{24}\right|
\end{array}\right.
$$

Therefore, solution is (25) which corresponds to (13).

- if $k_{1} k_{2}>0$ and $\left|k_{1}\right|>\left|k_{2}\right|$

$$
\left\{\begin{array}{l}
\left|y_{26}\right| \leq\left|x_{26}\right|=\left|z_{26}\right| \\
\left|x_{26}\right| \leq\left|x_{25}\right| \\
\left|z_{26}\right| \leq\left|z_{24}\right|
\end{array}\right.
$$

Therefore, solution is (26) which corresponds to (14).

\section{REFERENCES}

[1] N. Hogan, "Impedance control: An approach to manipulation: Part III-Applications," Journal of Dynamic Systems, Measurement, and Control, vol. 107, no. 1, pp. 17-24, Mar. 1985.

[2] S. Oh and Y. Hori, "Development of Two-Degree-of-Freedom control for robot manipulator with biarticular muscle torque," in American Control Conference, 2009.

[3] M. A. Lewis and T. J. Klein, "Achilles: A robot with realistic legs," IEEE Biomedical Circuits and Systems Conference (BIOCAS), Baltimore, 2008.

[4] F. Iida, J. Rummel, and A. Seyfarth, "Bipedal walking and running with spring-like biarticular muscles," Journal of Biomechanics, vol. 41, no. 3, p. 656-667, 2008.

[5] J. Dul, M. Townsend, R. Shiavi, and G. Johnson, "Muscular synergism-I. on criteria for load sharing between synergistic muscles," Journal of Biomechanics, vol. 17, no. 9, pp. 663-673, 1984.

[6] B. P. Yeo, "Investigations concerning the principle of minimal total muscular force," Journal of Biomechanics, vol. 9, 1976.

[7] K. Kaufman, K. An, W. Litchy, and E. Chao, "Physiological prediction of muscle forces-I. theoretical formulation," Neuroscience, vol. 40, no. 3, pp. 781-792, 1991.

[8] R. Happee and F. C. T. V. der Helm, "The control of shoulder muscles during goal directed movements, an inverse dynamic analysis," Journal of Biomechanics, vol. 28, no. 10, pp. 1179-1191, Oct. 1995.

[9] J. J. Collins, "The redundant nature of locomotor optimization laws," Journal of Biomechanics, vol. 28, no. 3, pp. 251-267, Mar. 1995.

[10] J. Cholewicki, S. M. McGill, and R. W. Norman, "Comparison of muscle forces and joint load from an optimization and EMG assisted lumbar spine model: Towards development of a hybrid approach,' Journal of Biomechanics, vol. 28, no. 3, pp. 321-331, Mar. 1995.

[11] J. M. Hollerbach and K. C. Suh, "Redundancy resolution of manipulators through torque optimization," IEEE Journal of Robotics and Automation, vol. 3, no. 4, p. 308-316, 1987.

[12] K. Yoshida, N. Hata, S. Oh, and Y. Hori, "Extended manipulability measure and application for robot arm equipped with bi-articular driving mechanism," in Industrial Electronics, 2009. IECON '09. 35th Annual Conference of IEEE, 2009, pp. 3083-3088.

[13] C. A. Klein and C. H. Huang, "Review of pseudoinverse control for use with kinematically redundant manipulators," IEEE Transactions on Systems, Man, and Cybernetics, vol. 13, p. 245-250, 1983.

[14] V. Salvucci, S. Oh, and Y. Hori, "Infinity norm approach for output force maximization of manipulators driven by bi-articular actuators," in Europe-Asia Congress on Mechatronics, EAM, 2010.

[15] S. Oh, Y. Kimura, and Y. Hori, "Reaction force control of robot manipulator based on biarticular muscle viscoelasticity control," in Advanced Intelligent Mechatronics (AIM), 2010 IEEE/ASME International Conference on, 2010, pp. 1105-1110.

[16] T. Tsuji, "A model of antagonistic triarticular muscle mechanism for lancelet robot," in Advanced Motion Control, 2010 11th IEEE International Workshop on, 2010, pp. 496-501.

[17] T. Fujikawa, T. Oshima, M. Kumamoto, and N. Yokoi, "Output force at the endpoint in human upper extremities and coordinating activities of each antagonistic pairs of muscles." Transactions of the Japan Society of Mechanical Engineers. C, vol. 65, no. 632, pp. 1557-1564, 1999.

[18] V. Salvucci, Y. Kimura, S. Oh, and Y. Hori, "BiWi: Bi-Articularly actuated and wire driven robot arm," in IEEE International Conference on Mechatronics (ICM), 2011. 